\title{
Rapid determination of general cell status, cell viability, and optimal harvest time in eukaryotic cell cultures by impedance flow cytometry
}

Christian Opitz ${ }^{1,}$, Grit Schade ${ }^{2, \$}$, Silvan Kaufmann², Marco Di Berardino², Marcel Ottiger ${ }^{2, *}$, Stephan Grzesiek ${ }^{1, *}$

${ }^{1}$ Focal Area Structural Biology and Biophysics, Biozentrum, University of Basel, CH-4056 Basel, Switzerland

2 Amphasys, Technopark Lucerne, CH-6039 Root D4, Switzerland

\$ Both authors contributed equally to the work

*Address correspondence to:

Stephan Grzesiek

Focal Area Structural Biology and Biophysics, Biozentrum

University of Basel, CH-4056 Basel, Switzerland

Phone: ++41612672100

FAX: $\quad++41612672109$

Email: Stephan.Grzesiek@unibas.ch

Marcel Ottiger

Amphasys

Technopark Lucerne

CH-6039 Root D4, Switzerland

Email: marcel.ottiger@amphasys.com

Keywords: Insect cells, yeast, viability, cell density, microfluidics, single cell, fermentation, bioprocessing, label-free, recombinant expression 


\begin{abstract}
The determination of cell viability is essential to many areas of life sciences and biotechnology. Typically, cell viability measurements are based on the optical analysis of stained cells, which requires additional labeling steps and is hard to implement on-line. Frequency-dependent impedance flow cytometry (IFC) provides a label-free, fast and reliable alternative to determine cell viability at the single-cell level based on the Coulter principle.

Here, we describe the application of IFC to eukaryotic cell cultures and compare the results to commonly used staining methods. Yeast cell parameters were assessed in normal and heatinactivated cells as well as in alcoholic fermentation and long-term batch cultures providing a precise and fast determination of the cell viability and further quantitative measures of the cell culture status. As an important new application we have investigated recombinant protein production in the widely used baculovirus insect cell expression system. The IFC analysis revealed the presence of a subpopulation of cells, which correlates with the protein expression yield, but is not detectable with conventional optical cell counters. We tentatively identify this subpopulation as cells in the late phase of infection. Their detection can serve as a predictor for the optimal time point of harvest. The IFC technique should be generally applicable to many eukaryotic cell cultures in suspension, possibly also implemented on-line.
\end{abstract}

\title{
Introduction
}

Cultivation of eukaryotic cell lines is essential to biological research and biotechnology, ranging from basic cell biological research over pharmaceutical and medical applications to food and biofuel production (Wei and Sommer 2013; Stewart 2017). Examples are highthroughput cellular screening applications, the production of recombinant proteins such as enzymes, hormones or antibodies, or the alcoholic fermentation by yeast. The cultivation typically involves preparation, seeding, growth, and harvesting of the cell cultures. All of these steps need to be carefully optimized for product yield, quality, and purity and properly controlled by monitoring suitable parameters.

Whereas the precise detection and control of physicochemical parameters such as $\mathrm{pH}$, temperature and oxygen is easy to achieve for a wide range of cell cultures, the accurate and efficient analysis of physiological cell parameters remains challenging. A prime cell parameter is the viability defined as the number of living cells relative to the total cell count. Cell viability is typically determined by semi-automated or automated counting of stained cells in wide-field light micrographs, which requires an additional labeling step, dedicated equipment, and is difficult to implement as an on-line or in situ method (Thomson et al. 2015; Cadena-Herrera et al. 2015). Methods for on-line analysis are e.g. 2D fluorometry, infrared spectroscopy and off- 
gas detection (Teixeira et al. 2009). However, these involve high instrument costs, multivariate calibration procedures and complex data analysis, and are so far not suited for single-cell analysis. A simpler on-line method relies on the determination of the total electrical permittivity of a cell suspension as an indicator for changes in cell size and membrane properties. The latter has been used to monitor the bulk status of baculovirus-infected insect cell cultures in bioreactors (Ansorge et al. 2007). A further label-free, on-line single-cell technology, Quantitative Phase Imaging (QPI) has emerged in the last couple of years (Barbau 2017). However, this approach is complex and seems currently only functional for animal cells, as studies on microorganisms have not been reported yet.

Impedance (micro-)flow cytometry (IFC) implemented on microfluidic chips presents an alternative non-invasive, label-free technique that can be used to rapidly characterize single cells in suspension on-line based on their dielectric properties (Cheung et al. 2010). Several variants of microfluidic chip designs have been described for cell analysis by IFC, which are all based on the same measurement principle (Di Berardino 2010): (i) Cells suspended in a conductive medium flow through a microchannel equipped with microelectrodes. (ii) An alternating (AC) field with adjustable frequency is applied over these electrodes and the complex impedance of the system is determined. (iii) The intrinsic impedance of the system with pure buffer flowing between the electrodes depends on the geometry of the electrodes and the microchannel, as well as the conductivity of the buffer. As suspended cells pass through the applied AC field, they change the detected impedance by their dielectric properties such as membrane capacitance and cytoplasmic conductivity, thereby reporting on cell size and morphology. (iv) These cell parameters can be specifically interrogated according to the chosen frequency of the applied AC field, where low $(0.1-0.5 \mathrm{MHz})$ and intermediate frequencies $(0.5-5 \mathrm{MHz})$ primarily provide information about cell volume and membrane properties, respectively, while higher frequencies (>5 MHz) rather probe intracellular structures and the cytoplasm (Cheung et al. 2010).

A particular technical advantage of IFC is the absence of optical elements, making the technology more robust than conventional flow cytometers, portable and therefore operational outside laboratories, without the need for adjustments and calibration. The development of compact IFC devices (Ampha ${ }^{\mathrm{TM}}$ Z32, Amphasys, Root, Switzerland) has now made this technology applicable for any kind of single-cell analysis. Its biggest potential lies in applications with a need for fast and label-free characterization of cell cultures. This is in particular the case for biotechnology applications, which typically rely on one specific and well characterized cell type with no need for complex multiparametric analyses using specific 
molecular markers. As an important commercial application, IFC is used for pollen quality analysis in the seed industry (Heidmann et al. 2016; Heidmann and Di Berardino 2017).

However, IFC can be used in many further areas of cell biology. Originally, IFC aimed at diagnostic applications in hematology, oncology or toxicology as a simpler and less expensive alternative to conventional fluorescence-based flow cytometry (Gawad et al. 2001; Gawad et al. 2004). Its potential has been demonstrated in many applications e.g. for the detection of cell viability, differentiation and apoptosis in cancer and stem cell research in the absence of specific cell markers (Pierzchalski et al. 2012; Crocetti et al. 2013; Crocetti et al. 2014) or for monitoring bovine erythrocyte parasite infection (Küttel et al. 2007) and the differentiation of fibroblasts into adipocytes (Schade-Kampmann et al. 2008). IFC has also been applied to yeast in an initial study demonstrating the separation of viable from dead yeast cells (Mernier et al. 2011) and to bacteria, as exemplarily shown for the determination of viability and membrane potential differences in Bacillus megaterium cells (David et al. 2012).

In this paper we demonstrate the suitability of IFC to routinely characterize yeast cultures in response to various treatments and to monitor the status of insects cells expressing recombinant proteins, thereby proving its potential for future on-line single-cell analysis applications. 


\section{Material and Methods}

\section{Single cell impedance measurements}

Frequency-dependent impedances of single cells in various cell suspensions were determined using an impedance flow cytometer (IFC) type Ampha ${ }^{\mathrm{TM}} \mathrm{Z} 32$ (Amphasys, Root, Switzerland). The measurements were performed at 0.5 and 10 or $12 \mathrm{MHz}$ (after initially screening from 0.5 to $20 \mathrm{MHz}$ ). The analyzed volume of cell suspensions was about $20 \mu \mathrm{l}$ containing $10^{5}-10^{7}$ cells per $\mathrm{ml}$. Impedances were usually recorded simultaneously at two to four frequencies. For each measurement, at least 10'000 to $30^{\prime} 000$ cells were analyzed at an event rate of 500 to 1 ' 000 cells/sec. Yeast cells were measured with a chip of $50 \mu \mathrm{m} \times 50 \mu \mathrm{m}$ channel size and modulation (M), amplification (A) and demodulation (D) levels were set a 5, 5 , and 1 , respectively with a trigger level of $0.013 \mathrm{~V}$. Insect cells were measured using a $30 \mu \mathrm{m}$ $\times 30 \mu \mathrm{m}$ channel size chip with modulation, amplification and demodulation levels set to 3, 6, and 1, respectively, and a trigger level of $0.1 \mathrm{~V}$ or $0.05 \mathrm{~V}$ (see below).

Initial tests showed that carry-over between consecutive samples separated by an air gap was less than $0.1 \%$. Therefore, the possible fully automated rinsing of the entire instrument fluidic system with deionized water was skipped to save time. Daily cleaning of the instrument with special cleaning solution was performed according to the manufacturer's protocol. Experimental data were collected and analyzed with the program AmphaSoft 2.1.2 (Amphasys, Root, Switzerland). For further analysis, phase and amplitude data were exported as csv-files and processed with R-Studio (v1.1.463).

\section{Yeast culture}

Yeast strain Scc ATCC 9080 was obtained from DMSZ GmbH (Braunschweig, Germany). Cells were first cultured overnight on malt agar plates (Sigma Aldrich, Buchs, Switzerland) at $30{ }^{\circ} \mathrm{C}$, and then single colonies were picked and incubated in YPD liquid medium (Sigma) at $30{ }^{\circ} \mathrm{C}$ with rotary shaking at $200 \mathrm{rpm}$ for maximally $24 \mathrm{~h}$ or as indicated in the text. A sample from the liquid culture at $\mathrm{t}=23 \mathrm{~h}$ (exponential growth phase) was split into two, and one half was heat-treated at $70^{\circ} \mathrm{C}$ for $15 \mathrm{~min}$ using a heat block for Eppendorf tubes. For IFC measurements, samples were diluted 1:20 in either (Fig. 1) the isosmotic IFC buffer AF6 (Amphasys, Switzerland) or (all other data) in 0.5x phosphate-buffered saline (PBS; Dulbecco's PBS, Sigma) and filtered using a $20 \mu \mathrm{m}$ filter (Sysmex CellTrics).

\section{Comparison with PI fluorescent staining of dead yeast cells}

Untreated and heat-treated samples as described before were mixed at predefined ratios (1:0, $3: 1,1: 1,1: 3,0: 1)$ to a volume of $100 \mu \mathrm{l}$ and further diluted to $2 \mathrm{ml}$ with AF6 and filtered using a $20 \mu \mathrm{m}$ filter. The samples were vortexed, and 20,000 cells were acquired in triplicates. A 
vertical gate at an impedance phase of about $235^{\circ}$ was used to separate between viable and dead cells. For viability determination by fluorescence microscopy, a $100 \mu 1$ of untreated/heat-treated cell mixtures as described above were diluted with $200 \mu \mathrm{l} \mathrm{AF6}$ and $100 \mu \mathrm{l} 3.0 \mu \mathrm{g} / \mathrm{ml}$ propidium iodide (PI, Sigma, Switzerland), and PI-stained (dead) and non-stained (viable) cells were counted using a fluorescence microscope (Leica). Linear regression analysis was performed between IFC- and fluorescence-determined viabilities. Standard deviations were calculated from three measurements for both methods.

\section{On-line measurement of long-term batch culture}

$S c c$ was cultured in $250 \mathrm{ml}$ Erlenmeyer flasks with $100 \mathrm{ml}$ YPD medium starting with a small aliquot of a fresh overnight culture for the indicated number of days in a shaking incubator at $28^{\circ} \mathrm{C}$. Samples were directly transferred without a filtration step from the culture to the impedance cytometer by a connected peristaltic pump. High cell concentrations $\left(>10^{7} \mathrm{cell} / \mathrm{s} / \mathrm{ml}\right)$ were diluted with fresh medium before measurement.

\section{On-line measurement of amphotericin-treated culture}

For amphotericin treatment, an exponentially growing $S c c$ culture in $100 \mathrm{ml}$ YPD medium was incubated with $1 \mu \mathrm{g} / \mathrm{ml}$ amphotericin B (Sigma) and further cultured for $5 \mathrm{~h}$ in a shaking incubator at $28{ }^{\circ} \mathrm{C}$. Samples were directly taken from the culture with a peristaltic pump connected to the impedance flow cytometer and measured without any filtration or dilution.

\section{Yeast fermentation in brewing process}

Top-fermenting Ale yeast (Saccharomyces cerevisiae) was sampled over 3 consecutive days from a draft beer fermentation at Doppelleu Boxer AG Switzerland, which was inoculated with yeast that had been used earlier for three consecutive fermentations. Wort was kept at a concentration of $9.93 \pm 0.08 \%(\mathrm{w} / \mathrm{v})$. After collection, samples were diluted 1:50 with $0.5 \mathrm{x}$ PBS, filtered using a $20 \mu \mathrm{m}$ filter (Sysmex CellTrics) and measured using the Ampha Z32 Impedance Flow Cytometer at frequencies of 2 and $12 \mathrm{MHz}$.

\section{Insect cell culture and infection}

General handling of Sf9 insect cell cultures was adapted from established protocols (O'Reilly et al. 1994). Cultures were grown in SF-4 medium (Bioconcept) at $27^{\circ} \mathrm{C}$ in $50 \mathrm{ml}$ bioreactor tubes (TPP) shaken at $250 \mathrm{rpm}$ (25 mm shaking diameter). Production of recombinant baculovirus (BV) for expression of trGFPuv (Khan 2003; Opitz et al. 2015; Franke et al. 2018) and subsequent infection of insect cells was based on the BV-infected insect cell (BIIC) stocks protocol (Wasilko et al. 2009). Prior to infection, cells were grown to a density of $1.5 \times 10^{6}$ cells $/ \mathrm{ml}$ in a $15 \mathrm{ml}$ volume and infected with $100 \mu \mathrm{l}$ of freshly prepared BIIC stocks. The optimal BIIC-to-cell ratio was determined by titration of small-scale cultures at identical 
conditions. To reduce ionic strength while preserving medium osmolarity, typically $200 \mu 1$ of insect cell culture were diluted with the same volume of $16 \%(\mathrm{w} / \mathrm{v})$ sucrose solution prior to IFC analysis.

\section{Quantification of trGFPuv expression}

Samples for fluorescence measurements were collected from insect cell cultures by centrifugation at $1,000 \mathrm{~g}\left(4^{\circ} \mathrm{C}\right)$ for $5 \mathrm{~min}$. Cell pellets and the corresponding supernatants were flash frozen in liquid nitrogen and stored at $-80{ }^{\circ} \mathrm{C}$ until further processing. Cells were resuspended in the same volume of $50 \mathrm{mM}$ TRIS buffer $(\mathrm{pH} 8)$ and lysed by sonication $(2 \times 15 \mathrm{~s}$, 0.5 duty cycle and $100 \%$ amplitude) using a VialTweeter (Hielscher). Lysates were cleared by centrifugation at $13,000 \mathrm{~g}\left(4^{\circ} \mathrm{C}\right)$ for $5 \mathrm{~min}$ and transferred into fresh tubes.

Fluorescence at $520 \mathrm{~nm}$ was measured following excitation at $397 \mathrm{~nm}$ using a NanoDrop ${ }^{\mathrm{TM}}$ 3300 spectrometer (Thermo Scientific). The final trGFPuv concentrations were determined by comparison to purified standards (Franke et al. 2018).

\section{Semi-automated microscopy image analysis of cell viability}

Wide-field microscopy semi-automated cell counting was performed using the Countess ${ }^{\mathrm{TM}}$ Automated Cell Counter system (Invitrogen) according to the manufacturer's protocol. Briefly, insect cell samples were mixed 1:1 (v/v) with a $0.4 \%$ trypan blue solution (Sigma) and subsequently loaded onto the counter chamber slides. The cell counter parameters for sensitivity, circularity, minimum and maximum cell size were set to $5,80 \%, 5 \mu \mathrm{m}$ and $60 \mu \mathrm{m}$, respectively.

\section{Optimization of trigger level for insect cell cultures}

The accuracy of IFC measurements can be improved by the proper adjustment of the amplitude trigger level, which rejects small non-cellular particles and noise via a software-based high-pass filter. Typical detected amplitudes are in the range of 0.5 to $2.5 \mathrm{~V}$ (see e.g. Fig. 5). An initial estimate of $0.1 \mathrm{~V}$ for the trigger level provided very consistent results for a trGFPuv expression culture until 72 hpi (Fig. S1). Thus this trigger level can be used for routine expressions, which are typically carried out until $\leq 72$ hpi. However, in the very late stage of infection ( $>78 \mathrm{hpi}$ ) an apparent increase in viability was detected using this trigger level (Fig. $\mathrm{S} 1$ and S2). This was traced to an underestimation of smaller lysed cells and cell debris with signal amplitudes below the trigger level. To counteract this effect, the trigger level was iteratively reduced to an optimal value of $0.05 \mathrm{~V}$, which then yielded a consistent decrease in cell viability until very late stages of the cell culture as expected for the progressing BV infection (Fig. 5 and 6). This trigger level was then used for all subsequent measurements of Sf9 cell cultures. 


\section{Results}

\section{IFC analysis of yeast cells}

Viability

To characterize the different behavior of viable and dead yeast cells by IFC, a Saccharomyces cerevisiae carlsbergensis (Scc) culture was grown overnight in yeast extract peptone dextrose (YPD) medium under standard conditions. Cells were then diluted in measurement buffer and analyzed directly. For the control experiment with dead cells an aliquot of the same sample was heat-treated before dilution and measurement. A frequency sweep from 0.5 to $20 \mathrm{MHz}$ revealed that 10 to $12 \mathrm{MHz}$ was optimal for the discrimination of viable and dead yeast cells (Fig. 1A). This frequency range was then kept for all subsequent experiments. The overlay of phase-amplitude scatter plots of viable and heat-treated (dead) Scc cells (Fig. 1A) shows that the impedances of heat-treated cells have significantly lower phases (x-axis) than those of untreated cells. This phase shift reflects the changed electrical properties of the cell membrane upon cell death, such as changes in conductivity and capacitance due to holes or inactivation of membrane pumps. The clear phase separation between viable and dead cells provided a very reproducible quantification of the viable cell fraction in mixed samples. Fig. 1B shows the comparison of viability determined by IFC and by manually counting propidium iodide (PI)-stained cells under a microscope for pre-defined mixtures of viable and dead Scc cells. The correlation between both data sets is very high $\left(r^{2}=0.996\right)$. Due to the robust, automated data analysis and the about two orders of magnitude higher number of analyzed cells (typically on the order of $10^{4}$ in less than one minute), the precision of the IFC data is also much higher than that of fluorescence microscopy, i.e. the average standard deviation of 3 independent viability measurements was $0.15 \%$ for IFC, but $4.4 \%$ for microscopy.

\section{On-line analysis of long-term aerobic batch cultures}

In contrast to the immediate cell death induced by heat or $70 \%$ ethanol, yeast cells show a different behavior during slow cell death in response to nutrient deficiency or low concentrations of toxic compounds. To analyze the influence of nutrient limitation and waste accumulation by IFC, $S c c$ cells were grown in a long-term aerobic batch culture without feeding. The culture was connected on-line to the impedance cytometer, and samples were taken automatically from the culture and measured. Fig. 2 shows a comparison of phase-amplitude scatter plots obtained from Scc cultures at day 1, 7 and 14 after inoculation of the culture, with two clearly distinguishable populations at day 7. A pronounced shift of the viable population to lower phase and smaller amplitude was detected over the full time course of 18 days (Fig. 2 and Fig. 3A). Cells at intermediate states, as seen on the right side of the stacked phase histograms (Fig. 3A) after 4 
days of culture, represent different metabolic, apoptotic or starving cell states. The histograms also indicate that the viable cells shift more or less gradually to lower phases over culture time, while the fraction of dead cells appears at day 2 and then becomes more and more pronounced. Fig. 3B shows the viable fraction of the yeast culture over time as quantified by the vertical line gate shown in Fig. 2 (vertical line), which separates the dead cells (left) from the viable cells (right).

\section{On-line analysis of an amphotericin-treated culture}

To illustrate cell death induced by cytotoxic compounds, Scc cells in the late exponential growth phase were exposed to $1 \mu \mathrm{g} / \mathrm{ml}$ of the antifungal drug amphotericin B and monitored by IFC. The culture was connected on-line to the impedance cytometer, and samples were taken every 10 minutes from the culture and measured directly. Similar to the starvation process (Fig. 3A) and to heat inactivation (Fig. 1A), a pronounced shift in the impedance phase of viable cells to lower values was observed (Fig. 3C). This process started at about 60 minutes after the addition of amphotericin and then continued over an intermediate state at 80 minutes to reach phase values corresponding to the dead population at 120 minutes.

\section{Yeast viability in a brewing process}

Growth conditions in a beer brewing fermenter are different from the previously discussed cases, as yeast cells produce ethanol during the fermentation process. Above a certain threshold, the increased ethanol concentration is toxic to yeast. As a proof of concept, we analyzed a brewer's yeast fermentation process, which had been started with a previously harvested yeast (Fig. 4). Until the end of day 1 (Fig. 4A, red), the yeast culture is in the late lag to early exponential ('log') phase with a fraction of dead cells from the earlier fermentation present. Substantial cell proliferation consistent with an exponential growth phase takes place from day 1 to day 2 (green), leading to an increase in cell concentration from 5.4 to $25.3 \times 10^{6}$ cells $/ \mathrm{ml}$, associated with an increase in cell viability from 57.1 to $83.9 \%$ (Fig. 4B). Until the end of day 3 , the viable population (blue) further shifts to lower phase angles while the growth in terms of cell concentration considerably slows down and the cell viability decreases to $72.6 \%$.

The ethanol concentration in the fermentation tank was only $0.1 \%$ until the end of day 1 after initiation of the fermentation process. Subsequently, it rapidly increased to $1.5 \%$ and $3.1 \%$ on days 2 and 3, respectively (Fig. 4B). The low production of alcohol during the first day can be attributed to the low cell concentration and reduced viability as well as the initial presence of oxygen. Only after all oxygen in the fermentation tank has been consumed, the yeast switches to the anaerobic ethanol fermentation. In later phases of the fermentation, the increased ethanol 
concentration is toxic to the yeast cells. Interestingly, these effects correlated with the observed pronounced phase shifts in IFC.

\section{IFC analysis of insect cells}

\section{Characterization of baculovirus-infected insect cells}

Recombinant protein production using baculovirus (BV)-infected insect cells requires tight monitoring of expression cultures to obtain the highest possible protein yields. We have used IFC to follow the kinetics of a BV infection of Sf9 insect cells. To correlate the kinetics with the protein expression, Sf9 cells were infected with a recombinant BV encoding the truncated cycle 3 mutant form of GFP (trGFPuv). The intrinsic fluorescence of trGFPuv was then used to quantify its expression in a fast and sensitive manner from the cell lysates without need of any purification steps.

An initial IFC analysis of insect cell cultures was carried out 48 hours post infection (hpi). This time corresponds to the late phase of BV infection and is close to the optimal harvesting point with regard to recombinant protein yield. A wide range of frequencies was tested and optimal separation of distinct cell populations was found at $0.5 \mathrm{MHz}$ (Fig. 5, Fig. S4). Further instrument parameters (i.e. trigger level) were then iteratively adjusted for optimal detection of cells and rejection of cell debris (see Materials and Methods). At a frequency of $0.5 \mathrm{MHz}$, variations in the impedance strongly correlate to changes in cell size and to a minor extent to changes in membrane structure (Gawad et al. 2004). Such changes are expected during the progression of the BV infection, which causes cell swelling and an increasing vacuolization of Sf9 cells (O'Reilly et al. 1994).

A visual inspection of the IFC phase-amplitude scatter plots during the full time course of the BV infection from 0 to 99 hpi reveals three distinct regions (Fig. 5). Analogous to yeast (Fig. $1)$, the well separated region at higher phase values $\left(150^{\circ}\right.$ to $\left.220^{\circ}\right)$ was identified with the 'viable' cell population and separated in the analysis software from the remaining fraction by vertical gating. This viable cell population initially increases its impedance amplitude by about $60 \%$ from 0 to $48 \mathrm{hpi}$, corresponding to the swelling of cells after infection. The shift in the impedance amplitude thus provides a quantitative readout of the infection level, which can be used to determine the optimal virus to cell ratio (multiplicity of infection, MOI) from virus titration assays. After 48 hpi, the impedance amplitude of the viable cells decreases (cells shrink) and their population gradually vanishes until 99 hpi (see also Fig. 6).

The remaining area at lower phase values $\left(110^{\circ}\right.$ to $\left.150^{\circ}\right)$ was further subdivided into two distinctive regions by polygon gates (Fig. 5). The first polygon region at $\sim 110^{\circ}$ to $135^{\circ}$ was assigned to 'dead' cells, whereas the second polygon region at $\sim 135^{\circ}$ to $150^{\circ}$ was tentatively 
assigned to 'late phase of infection (LPI)' cells. The initially very small dead cell population gradually increases until $48 \mathrm{hpi}$ and then rapidly dominates after $60 \mathrm{hpi}$, in agreement with their identification as dead cells (Fig. 5, 6). The population assigned to LPI cells initially increases up to 60 hpi and subsequently rapidly disappears possibly due to cell lysis in the very late stage of infection.

\section{Comparison of IFC to image analysis}

To compare IFC and image analysis for their ability to predict the optimal harvest time (Fig. 6), we determined the cell viability by the Countess ${ }^{\mathrm{TM}}$ imaging cell counter using trypan blue and simultaneously quantified individual cell populations by IFC using the indicated gates (Fig. 5) for a total of $99 \mathrm{hpi}$.

Following infection, the viability determined by image analysis decreased first mildly from an initial $\sim 100 \%$ to $\sim 80 \%$ until $60 \mathrm{hpi}$, and then strongly to $42 \%$ at 99 hpi (Fig. 6, blue line). However, while the population of viable cells as detected by IFC was similar until $24 \mathrm{hpi}$, it much more rapidly decreased thereafter, reaching $50 \%$ at $\sim 60$ hpi and $5 \%$ at 96 hpi. A very pronounced difference between image analysis and IFC detection is evident at 79 hpi, where viability values are $78 \%$ and $11 \%$, respectively. At this point, the IFC phase-amplitude scatter plot clearly shows $87 \%$ of cells in the gating region assigned to dead cells. Apparently, image analysis based on trypan blue staining fails to detect the massive reorganization of cells that is indicated by the changes in cellular impedance. These massive changes in cellular structure agree with the nuclear fragmentation and blebbing of cells into apoptotic bodies, which are expected during the BV-induced apoptosis and the concomitant extensive BV replication (O'Reilly et al. 1994).

Image analysis not only assesses cell viability, but also provides the cell size, which varies significantly during the distinct phases of the BV infection (O'Reilly et al. 1994). As such it is typically used as a reporter for the status and quality of the infection. Mean sizes of the viable cells obtained at different time points during BV infection by the Countess ${ }^{\mathrm{TM}}$ imaging cell counter showed a swelling of the infected cells from an initial $12.8 \mu \mathrm{m}$ to a plateau around $16 \mu \mathrm{m}$ between 23 and $60 \mathrm{hpi}$, followed by a final decrease to $8.5 \mu \mathrm{m}$ at $99 \mathrm{hpi}$ (Fig. 7a, dashed red line). A very similar trend was found in IFC mean amplitude (Fig. 7a, dashed black line) of the viable population as defined in Fig. 5, albeit with a steeper increase between 7 and 23 hpi and more rapid decrease starting at 60 hpi. A comparison of image and IFC analysis at each time point, revealed a very good correlation $\left(r^{2}=0.901\right)$ of the amplitude data with the mean cell sizes (Fig. 7b). Thus the mean IFC amplitude of viable cells also provides a measure of the cell size and comparable information to image analysis on the status and quality of the infection. 


\section{Subpopulation size correlates with recombinant protein production}

As compared to image analysis, IFC further discriminates the non-viable population into the previously described late phase of infection (LPI) and dead fractions. Whereas the dead fraction continuously increases, the LPI fraction builds up from 48 to $60 \mathrm{hpi}$ and disappears until $72 \mathrm{hpi}$. Remarkably, the size of the LPI population correlates with the concentration of expressed trGFPuv detected in the lysed cell pellet (Fig. 6, dotted red and solid black lines) peaking between 48 and 60 hpi.

As the BV infection progresses further, cells are lysed and the expressed trGFPuv is also released into the medium. This extracellular fraction of trGFPuv was quantified from the culture supernatant (Fig. 6, dashed black line). It correlates exactly with the fraction of the dead cell population determined by IFC (Fig. 6, dashed red line).

Albeit the extracellular amount of expressed trGFPuv eventually exceeds the intracellular trGFPuv, unwanted reactions in the culture medium and proteases released from the lysed cells may degrade the quality of the expressed protein. Thus it is often not desirable to use this released protein fraction for subsequent applications. Since the LPI population detected by IFC correlates with the expressed intracellular protein, its detection is a good predictor for the optimal harvest time of intracellular protein.

\section{Long term IFC analysis of maintenance cultures}

To monitor the viability of continuously growing, non-infected Sf9 maintenance cultures, we have followed their growth over $99 \mathrm{~h}$ after initial passage by IFC (Fig. S3). The percentage of viable cells $(97.9 \% \pm 0.7 \%)$ remained constant for 79 hpi of continuous growth. Only when the typical subculturing interval was exceeded at $99 \mathrm{hpi}$, a decrease in cell viability to $\sim 93.1 \%$ occurred, which was possibly due to nutrient limitation or the very high cell density. A more detailed inspection of the IFC amplitude distribution, which correlates to cell size, reveals that the initially wide size distribution narrows during the time from 0 to $60 \mathrm{~h}$. In contrast to the infected cultures, a significant fraction of LPI or dead cells was not detected until $60 \mathrm{~h}$, but slightly increased at $99 \mathrm{~h}$.

\section{Discussion}

In this study we have demonstrated that the label-free IFC single cell analysis is well suited for the characterization of cell cultures using several yeast and insect cell models as examples. The IFC analysis can be carried out off-line and on-line, enabling real-time monitoring of prolonged fed-batch cultures in bioreactors. IFC not only provides cell count and viability information, but also data about status and morphological changes in response to external factors like nutrient limitation, toxic substances, or infection. 
So far, impedance-based cell analysis has predominantly been performed with conventional Coulter counters, which operate at DC or low-frequency AC and provide mainly cell count and cell size information. Viability determination with these devices is only obtained indirectly as an apparent reduction of cell volume, which occurs for cells without a rigid cell wall. For such cells, the membrane stops to act as an electrical barrier upon death, and as a consequence the detected volume is reduced to the nucleus (Schott et al. 2015). This is the case for animal cells, but not for microorganisms like bacteria and yeast, which have a rigid cell wall and barely shrink when they die. For bacteria, which do not have cell nuclei, Coulter counters definitely cannot be used to determine the dead/viable cell ratio, whereas IFC is well suited to determine both viability and membrane potential of such cells (David et al. 2012).

It has been reported that the viability of yeast cells can be assessed to a certain extent from their size alone using Coulter counters (Tibayrenc et al. 2010). However, the discriminative power is much lower than for IFC, which yields additional information from the frequencydependent membrane capacitance and cytoplasmic conductivity. In fact, the shrinking in cell size indicated by the change in average impedance amplitudes of the long-term aerobic batch culture from day 1 to day 7 (Fig. 2) is significantly larger than that from day 7 to the dead yeast cells at day 17. These observations indicate that the yeast cells in the initial lag phase grow in size before they start to divide and then maintain a smaller average size, which is similar to the size of dead cells. An evaluation of viability only on the basis of size information thus seems to be rather misleading. IFC however not only provides a clear distinction between dead or living cells, it also indicates different stages of the viable cell population during the time course of the yeast cultivation.

The results obtained on yeast cultures treated with the fungicide amphotericin B show that IFC can be used for label-free toxicological studies of cells in suspension, e.g. for determination of $\mathrm{IC}_{50}$ values. It seems obvious that such toxicological studies can be extended to mammalian cells, for which size information is expected to further increase the accuracy of the IFC cell characterization, as in many cases apoptotic and dead mammalian cells are significantly smaller than viable cells (Elmore 2007). Such analyses have so far been accessible mainly by Electric Cell-substrate Impedance Sensing (ECIS), which is limited to adherently growing cells and provides results at the culture, but not at the single-cell level (Wegener et al. 2000).

In beer brewing, yeast is usually obtained from propagation tanks or harvested and reused from a previous fermentation. While propagated yeast usually has a very high viability ( $>95 \%)$, the viability of harvested yeast can be lower due to the alcohol content at the end of the previous fermentation, storage and the repetitive use. To demonstrate the clear distinction by IFC between 
viable and dead cells during the early stage of fermentation, a beer fermentation starting with low-viability harvest yeast as input material (57.1\%, usually between $75 \%$ and $90 \%$ ) has been presented here. Besides monitoring cell viability throughout the entire fermentation, IFC provides valuable information about the status of the cell culture, such as the pronounced shift of viable cells towards lower phase values, which coincides with the transition from the exponential to the stationary growth phase. This extended status information may be of particular value during fermentation trials.

For insect cell cultures, IFC proved well suited to not only determine reliably the cell viability, but also to estimate changes of cell size. The latter can be used to assess the goodness of the BV infection in a similar way as obtained from conventional image analysis. Extensions to many other animal cells in suspension are obvious. Remarkably, the IFC analysis of BVinfected insect cells revealed a more rapid alteration of cellular properties towards the end of the infection phase as compared to the conventional trypan blue staining microscopy. This was accompanied by the appearance of a distinct cell population, which we tentatively identified with cells in the late phase of infection undergoing a pronounced reorganization of their membrane. The appearance of this population correlated with the amount of recombinantly expressed intracellular protein. Thus IFC can not only be used to screen for optimal expression conditions and virus titer, but also to identify the best time for the harvest of intracellular protein in the BV insect cell system.

Besides the described gain in information on cellular properties, IFC has considerable practical advantages. It does not require labeling, which constitutes an additional effort and can be a significant source of error. In contrast to image analysis, IFC is not affected by manual settings such as focal area or small sample sizes, which have a strong impact on the accuracy and precision of the cell size and viability measurements. IFC is rather tolerant towards buffer conditions as the analysis can usually be performed directly in the culture medium such that no additional time for buffer exchange is required. Suitable conditions range between $10^{4}$ and $10^{7}$ cells $/ \mathrm{ml}$ in buffers with conductivities between 2 and $10 \mathrm{mS} / \mathrm{cm}$, compatible with many cell cultures. For too high cell or ion concentrations, a simple dilution of the sample with a suitable buffer is usually sufficient. Thus in many cases IFC can be implemented easily as an on-line method. IFC chips are incompatible with solutions containing more than $5 \%$ of organic solvents, which are rarely encountered in routine cell culture applications. Very high cell concentrations, chain forming cells and large impurities may increase the risk of clogging the microfluidic chip. As countermeasure, large impurities can be removed by filters, whereas chips 
with channel diameters up to $250 \mu \mathrm{m}$ may be used for chain forming cells. No clogging was observed in the present study.

In summary, we have shown that IFC is a rapid, robust, and label-free technology for the viability determination of microbial and animal cell cultures. Valuable additional information on membrane capacitance and cytoplasmic conductivity is contained in the frequencydependent complex impedance that can be used to further characterize cellular activities during fermentation and to predict optimal harvest times. 


\section{Acknowledgement}

We thank Alexandra Meng for valuable suggestions on the manuscript and Doppelleu Boxer AG for providing yeast samples from their large-scale production tank.

\section{Notes}

Author contributions

Christian Opitz, Grit Schade, Marcel Ottiger and Stephan Grzesiek designed the study. Christian Opitz, Grit Schade and Silvan Kaufmann carried out data acquisition, analysis and interpretation. Marco Di Berardino, Marcel Ottiger and Stephan Grzesiek participated in data interpretation. Christian Opitz, Grit Schade, Silvan Kaufmann, Marco Di Berardino, Marcel Ottiger and Stephan Grzesiek wrote the manuscript.

\section{Funding information}

This work was supported by the Swiss National Science Foundation (grant 31-173089 to S.G.).

Compliance with ethical standards

Conflict of interest

Grit Schade, Silvan Kaufmann, Marco Di Berardino and Marcel Ottiger are employees of Amphasys AG, Root, Switzerland. Christian Opitz and Stephan Grzesiek declare that they have no conflict of interest.

\section{Ethical approval}

This article does not contain any studies with human participants or animals performed by any of the authors. 


\section{References}

Ansorge S, Esteban G, Schmid G (2007) On-line monitoring of infected Sf-9 insect cell cultures by scanning permittivity measurements and comparison with off-line biovolume measurements. Cytotechnology 55:115124. doi: $10.1007 / \mathrm{s} 10616-007-9093-0$

Barbau J (2017) Continuous suspension cell culture monitoring in bioreactors using quantitative phase imaging. $1-1$.

Cadena-Herrera D, Esparza-De Lara JE, Ramírez-Ibañez ND, López-Morales CA, Pérez NO, Flores-Ortiz LF, Medina-Rivero E (2015) Validation of three viable-cell counting methods: Manual, semi-automated, and automated. Biotechnol Rep (Amst) 7:9-16. doi: 10.1016/j.btre.2015.04.004

Cheung KC, Di Berardino M, Schade-Kampmann G, Hebeisen M, Pierzchalski A, Bocsi J, Mittag A, Tárnok A (2010) Microfluidic impedance-based flow cytometry. Cytometry A 77:648-666. doi: 10.1002/cyto.a.20910

Crocetti S, Beyer C, Schade G, Egli M, Fröhlich J, Franco-Obregón A (2013) Low intensity and frequency pulsed electromagnetic fields selectively impair breast cancer cell viability. PLoS One 8:e72944. doi: 10.1371/journal.pone.0072944

Crocetti S, Beyer C, Unternährer S, Benavides Damm T, Schade-Kampmann G, Hebeisen M, Di Berardino M, Fröhlich J, Franco-Obregón A (2014) Impedance flow cytometry gauges proliferative capacity by detecting TRPC1 expression. Cytometry A 85:525-536. doi: 10.1002/cyto.a.22461

David F, Hebeisen M, Schade G, Franco-Lara E, Di Berardino M (2012) Viability and membrane potential analysis of Bacillus megaterium cells by impedance flow cytometry. Biotechnol Bioeng 109:483-492. doi: $10.1002 /$ bit.23345

Di Berardino M (2010) The Microflow Cytometer. In: Ligler FS, Kim JS (eds) Electrical Detection in Microfluidic Flow Cytometers. Pan Stanford Publishing, p 379

Elmore S (2007) Apoptosis: a review of programmed cell death. Toxicol Pathol 35:495-516. doi: $10.1080 / 01926230701320337$

Franke B, Opitz C, Isogai S, Grahl A, Delgado L, Gossert AD, Grzesiek S (2018) Production of isotope-labeled proteins in insect cells for NMR. J Biomol NMR 1-12. doi: 10.1007/s10858-018-0172-7

Gawad S, Cheung K, Seger U, Bertsch A, Renaud P (2004) Dielectric spectroscopy in a micromachined flow cytometer: theoretical and practical considerations. Lab Chip 4:241-251. doi: 10.1039/b313761a

Gawad S, Schild L, Renaud P (2001) Micromachined impedance spectroscopy flow cytometer for cell analysis and particle sizing. Lab Chip 1:76-82. doi: 10.1039/b103933b

Heidmann I, Di Berardino M (2017) Impedance Flow Cytometry as a Tool to Analyze Microspore and Pollen Quality. Methods Mol Biol 1669:339-354. doi: 10.1007/978-1-4939-7286-9_25

Heidmann I, Schade-Kampmann G, Lambalk J, Ottiger M, Di Berardino M (2016) Impedance Flow Cytometry: A Novel Technique in Pollen Analysis. PLoS One 11:e0165531. doi: 10.1371/journal.pone.0165531

Khan F (2003) Letter to the Editor: 1H, 15N and 13C backbone assignment of the Green Fluorescent Protein (GFP). J Biomol NMR 26:281-282. doi: 10.1023/A:1023817001154

Küttel C, Nascimento E, Demierre N, Silva T, Braschler T, Renaud P, Oliva AG (2007) Label-free detection of Babesia bovis infected red blood cells using impedance spectroscopy on a microfabricated flow cytometer. Acta Tropica 102:63-68. doi: 10.1016/j.actatropica.2007.03.002

Mernier G, Piacentini N, Tornay R, Buffi N, Renaud P (2011) Cell viability assessment by flow cytometry using yeast as cell model. Sensors and Actuators B: Chemical 154:160-163-163. doi: 10.1016/j.snb.2009.11.066

O'Reilly DR, Miller LK, Luckow VA (1994) Baculovirus expression vectors: a laboratory manual. Oxford University Press 
Opitz C, Isogai S, Grzesiek S (2015) An economic approach to efficient isotope labeling in insect cells using homemade 15N-, 13C- and 2H-labeled yeast extracts. J Biomol NMR 62:373-385. doi: 10.1007/s10858015-9954-3

Pierzchalski A, Hebeisen M, Mittag A, Bocsi J, Di Berardino M, Tárnok A (2012) Label-free hybridoma cell culture quality control by a chip-based impedance flow cytometer. Lab Chip 12:4533-4543. doi: $10.1039 / \mathrm{c} 21 \mathrm{c} 40408 \mathrm{~g}$

Schade-Kampmann G, Huwiler A, Hebeisen M, Hessler T, Di Berardino M (2008) On-chip non-invasive and label-free cell discrimination by impedance spectroscopy. Cell Prolif 41:830-840. doi: 10.1111/j.13652184.2008.00548.x

Schott L, Sommer C, Wittek J, Myagmar K, Walther T, Baßler M (2015) Cell size discrimination based on the measurement of the equilibrium velocity in rectangular microchannels. Micromachines 6:634-647. doi: $10.3390 / \operatorname{mi} 6050634$

Stewart GG (2017) Yeast Viability and Vitality. In: Brewing and Distilling Yeasts. Springer, pp 147-165

Teixeira AP, Oliveira R, Alves PM, Carrondo MJT (2009) Advances in on-line monitoring and control of mammalian cell cultures: Supporting the PAT initiative. Biotechnol Adv 27:726-732. doi: 10.1016/j.biotechadv.2009.05.003

Thomson K, Bhat A, Carvell J (2015) Comparison of a new digital imaging technique for yeast cell counting and viability assessments with traditional methods. Journal of the Institute of Brewing 121:231-237. doi: $10.1002 /$ jib. 224

Tibayrenc P, Preziosi-Belloy L, Roger J-M, Ghommidh C (2010) Assessing yeast viability from cell size measurements? J Biotechnol 149:74-80. doi: 10.1016/j.jbiotec.2010.06.019

Wasilko DJ, Lee SE, Stutzman-Engwall KJ, Reitz BA, Emmons TL, Mathis KJ, Bienkowski MJ, Tomasselli AG, Fischer HD (2009) The titerless infected-cells preservation and scale-up (TIPS) method for large-scale production of NO-sensitive human soluble guanylate cyclase (sGC) from insect cells infected with recombinant baculovirus. Protein Expr Purif 65:122-132. doi: 10.1016/j.pep.2009.01.002

Wegener J, Keese CR, Giaever I (2000) Electric cell-substrate impedance sensing (ECIS) as a noninvasive means to monitor the kinetics of cell spreading to artificial surfaces. Exp Cell Res 259:158-166. doi: 10.1006/excr.2000.4919

Wei N, Sommer B (2013) Chapter 7: Cell Viabilty Measurements. In: Flickinger MC (ed) Upstream Industrial Biotechnology, Volume Set. Wiley, p 1854 


\section{Figure Legends}

Figure 1: Discrimination of viable and dead yeast cells by impedance flow cytometry (IFC) and comparison with fluorescence microscopy (FCM). A) Overlay of IFC phase (x-axis) and amplitude (y-axis) scatter plots at $10 \mathrm{MHz}$ frequency of viable (untreated; green dots) and dead (heat-treated; red dots) Scc cells with adjunct histograms. B) Mixed samples of viable and dead (heat-treated) Scc cells were analyzed for viability by IFC and FCM. For IFC quantification of dead and viable cells in phase-amplitude scatter plots, the vertical gating as shown in Fig. 2 was used. Viable-cell fractions of IFC and FCM measurements were correlated by linear regression analysis. Standard deviations (shown by error bars) were determined from three measurements of each sample with both techniques. For IFC, the errors are in the range of $0.15 \%$ and therefore not visible in the diagram.

Figure 2: On-line analysis of long-term Scc batch culture. Phase-amplitude scatter plots of yeast culture samples taken at day 1, 7 and 14 after culture initiation. The black vertical lines mark the border between low phase/dead and high phase/viable cells.

Figure 3: Viability analysis of long-term $S c c$ batch culture and amphotericin-treated $S c c$ culture. A) Stacked phase histograms of impedance analysis at $10 \mathrm{MHz}$ of Scc long-term batch culture with indicated culture times. B) Cell viability of long-term batch yeast culture. Values are based on vertical gating (black line) as shown in Fig. 2. C) Impedance analysis at $10 \mathrm{MHz}$ of $S c c$ culture containing $1 \mu \mathrm{g} / \mathrm{ml}$ amphotericin. Stacked phase histograms are shown from samples taken at time points between $0 \mathrm{~h}$ and $5 \mathrm{~h}$ after addition of amphotericin.

Figure 4: Yeast fermentation in beer brewing process. A) Overlay of phase-amplitude scatter plots of a fermenting yeast culture in a draft beer production process sampled over 3 days after process start. Sampling time points are indicated. The yeast for this fermentation was obtained from the last harvest of three previous consecutive fermentation processes. B) Cell density (black), viability (orange), and ethanol concentration (blue) of the yeast fermentation shown in panel $\mathrm{A}$ as a function of the time after the process start.

Figure 5: Impedance analysis of baculovirus-infected Sf9 insect cells. Phase-amplitude data at $0.5 \mathrm{MHz}$ were obtained at discrete time points indicated as hours post infection (hpi) with the baculovirus ( 0 hpi presents the reference culture prior to infection). The vertical black line indicates the separation of viable (high phase) and non-viable (low phase) cell populations with 
the latter region being further separated into late phase of infection (LPI) and dead cells using two polygon gates as indicated.

Figure 6: Time-resolved analysis of viability and expression kinetics of baculovirusinfected Sf9 cells. Cultures infected with recombinant baculovirus encoding the trGFPuv gene were sampled at discrete time points following infection as indicated by hpi. Cell viability was analyzed by image analysis (Countess) and IFC using a trigger level of $0.05 \mathrm{~V}$. For the latter, populations of viable, dead, and late phase of infection (LPI) cells were determined by separating the phase-amplitude data into three distinct regions as shown in Figure 5. Intra- and extracellular trGFPuv concentrations were determined by fluorescence measurements of the lysed cell pellet or the culture supernatant, respectively.

Figure 7: Changes in sizes and IFC amplitudes of baculovirus-infected Sf9 cells. A) Cultures infected with recombinant baculovirus encoding the trGFPuv gene were sampled at discrete time points following infection as indicated by hpi. Mean sizes of viable cells were directly determined by image analysis using the default instrument settings. Mean amplitudes of viable cells were obtained by calculating the mean of the amplitude data selected for the viable population indicated in Figure 5 using IFC. B) Correlation of mean viable cell amplitude determined by IFC with the mean viable cell size obtained from conventional image analysis (data as A). The dashed black line indicates a linear fit of all points with Mean viable cell size $[\mu \mathrm{m}]=6.68$ Mean viable cell amplitude $(\mathrm{IFC})[\mathrm{AU}]+9.91(N=9)$. The error bars show the standard error of the mean obtained from measurements of three independent cell cultures. 


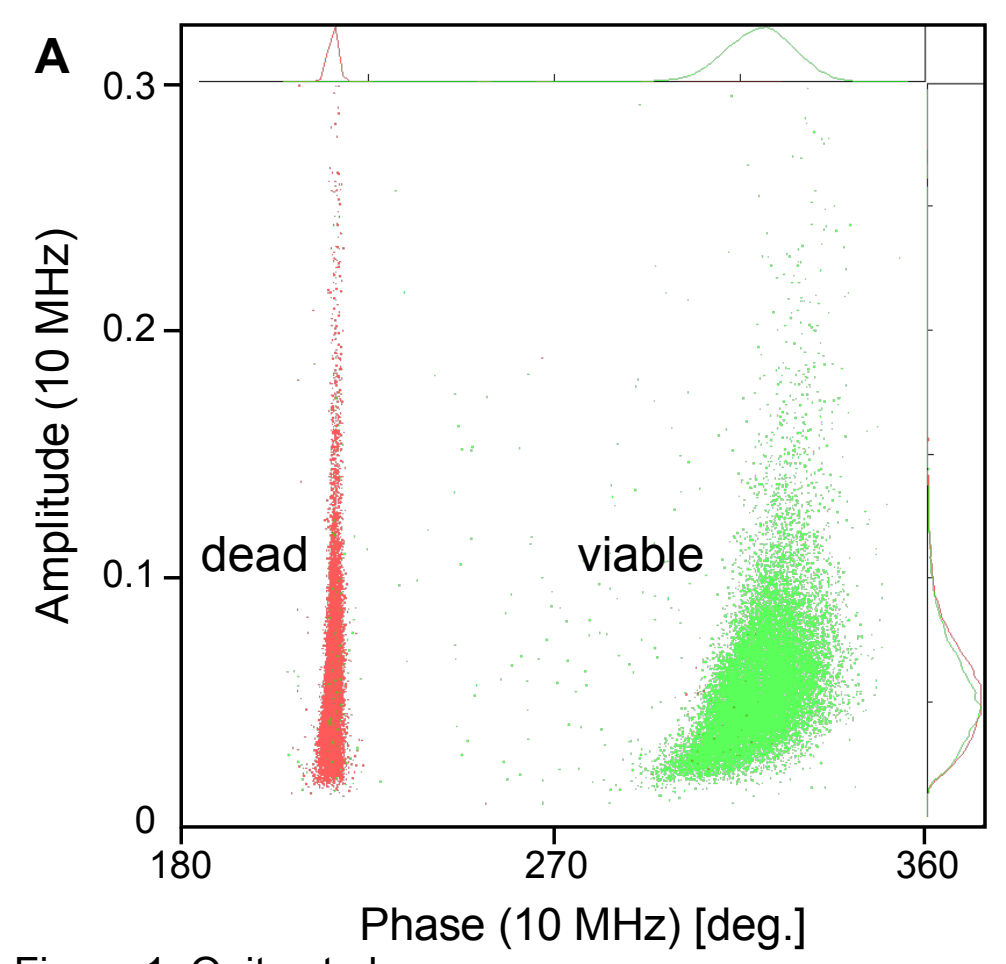

Figure 1, Opitz et al.

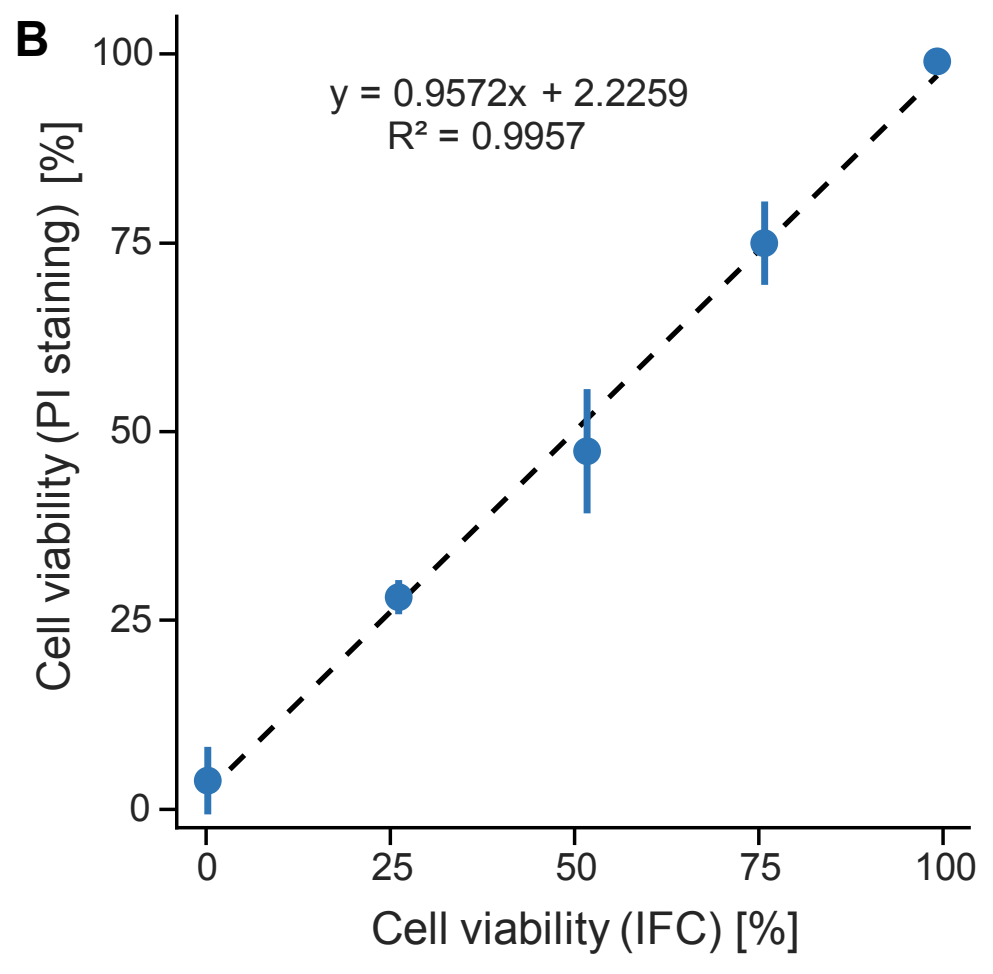




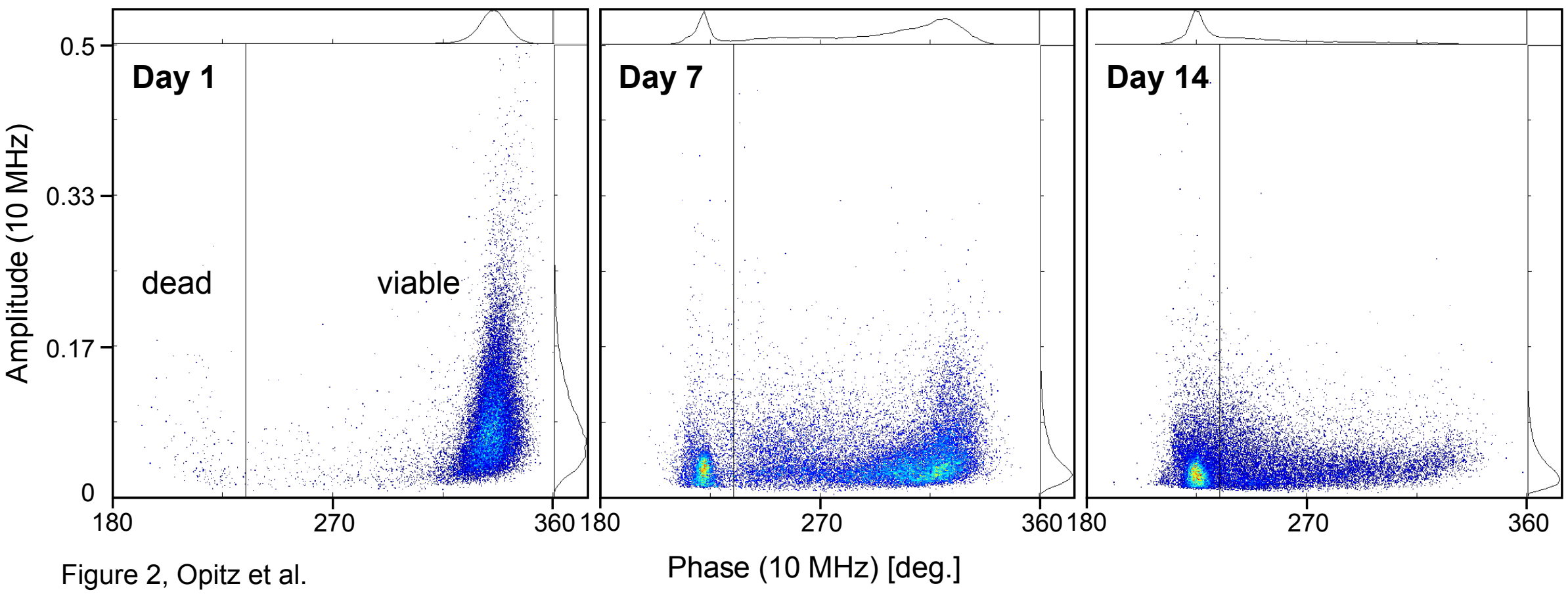




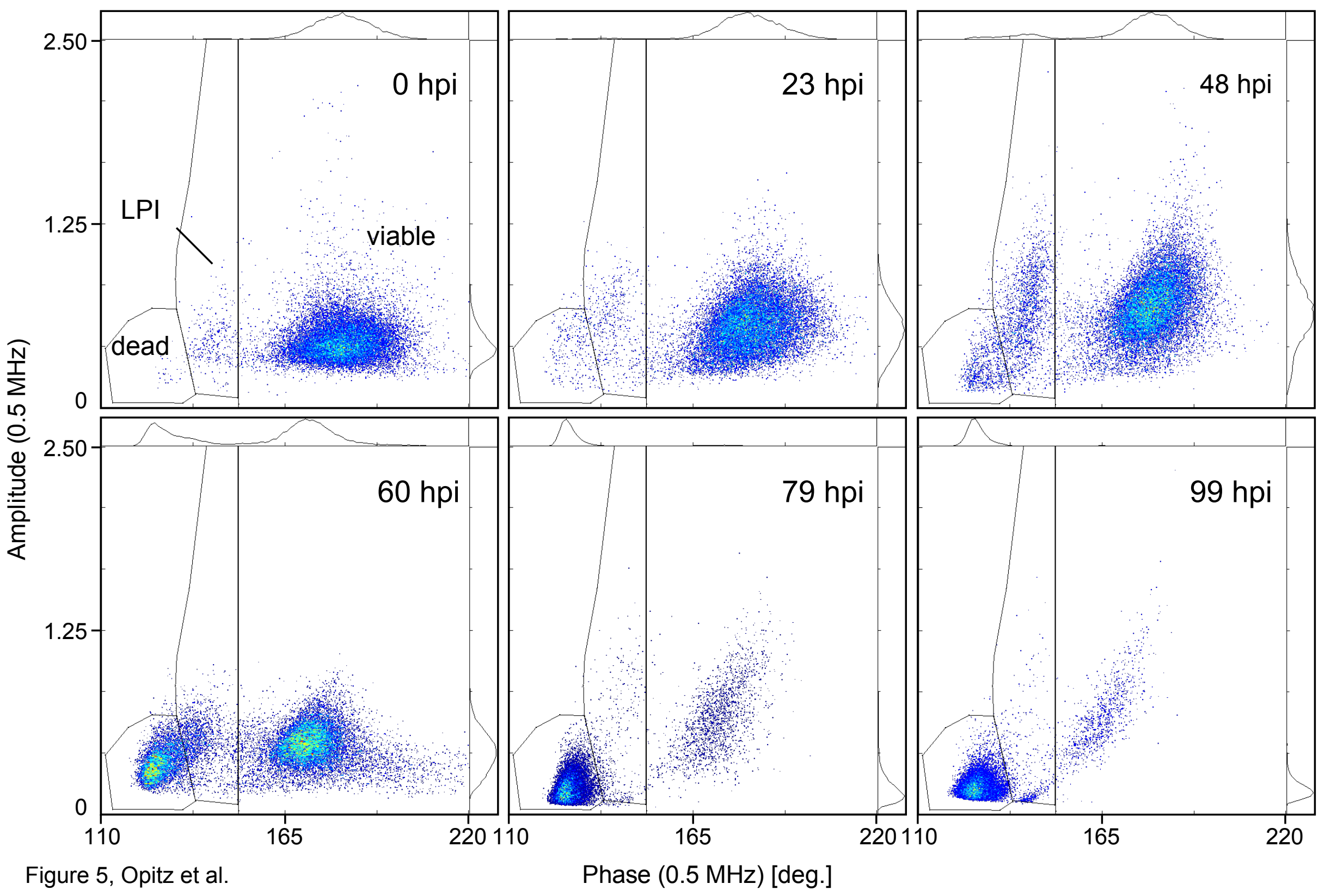


